\title{
Modeling, Analysis \& Implementation of Improved AODV Routing Protocol in MANETs
}

\author{
Mahesh Kumar Yadav \\ Research Scholar, NIMS University, \\ Jaipur, Rajasthan \\ INDIA
}

\author{
Ram Kishan Khola \\ Prof. \& Head, Deptt.of ECE, \\ P.D.M College of Engg, \\ Haryana, INDIA
}

\author{
Deepak Dembla \\ Asso.Professor, Deptt. of CSE, \\ AIET, Jaipur, Rajasthan \\ INDIA
}

\begin{abstract}
Network topology can change quickly and unpredictably in mobile adhoc networks (MANETs), since there may exist a large number of independent ad hoc connections. All the routing protocols use a basic technique of route request transmission i.e. blind flooding which causes a lot of overheads in terms of end to end delay, packet delivery ratio, no. of routing packets generated etc. Because this is a bottleneck in performance of AODV protocol, an improved AODV protocol is proposed here. The proposed protocol is modeled, analyzed and implemented on base AODV routing protocol, based on improved method of route discovery and the proposed protocol adjusts itself dynamically based on the network density of MANET. The proposed protocol implementation is done in GloMoSim. The simulation results show that improved-AODV (I-AODV) protocol significantly reduces the no. of rebroadcasts and hence reduces the routing overheads caused due to broadcast storm in the network.
\end{abstract}

Keywords- MANET, AODV, broadcast, MANETs, GloMoSim, collision rate, mobility, route discovery.

\section{INTRODUCTION}

A MANET [1] is a self-configuring network that can have an arbitrary topology along the time. Each mobile host works as a router and it is free to move randomly and connect to other hosts arbitrarily. MANET consists of a set of wireless nodes, which are spread over a geographical area. These nodes are able to perform processing as well as capable of communicating with each other by means of a wireless ad hoc network. Routing protocols can be primarily categorized as proactive (table-driven) and reactive (on-demand) protocols. Table driven protocols attempts to maintain consistent up-todate information from each node to every other node in the network. Proactive routing protocols maintain routes between all pairs of nodes at all times. Reactive routing protocols, on the other hand, do not maintain routes. When a route is needed, they will initiate route discovery process to find a route (or possibly multiple routes). Only after the route is found, the node can start the communication.

Several routing algorithms for MANETs have been proposed in the literature such as ad hoc on-demand distance vector routing [2],[1] dynamic source routing protocol (DSR)[3] optimized link state routing protocol(OLSR).

Broadcasting is a basic communication technique of for route discovery in MANETs and its basic mechanism is known as pure or blind flooding which results in serious contention, collisions and redundancy in the network, called as broadcast storm problem[4]. To remove these problems several algorithms are proposed to reduce the number of retransmissions and to improve the network performance. The simplest and most trivial broadcasting algorithm is pure flooding. Every node that receives the broadcast message retransmits it to all its neighbors. The problem of pure flooding is that it produces many redundant messages, which may consume scarce radio and energy resources, and cause collision that is called broadcast storm problem. Therefore, the basic principle of designing an efficient and resource conservative broadcast algorithm is trying to reduce the redundant messages[5], which means to inhibit some nodes from rebroadcasting and the message can still be disseminated to all nodes in the network.

In this research paper, an improved method of route discovery is implemented in which each intermediate node forwards the RREQ packet to its neighbor with some probability, based on the density of neighborhood nodes. In this approach, neighborhood densities are divided in two categories; dense and sparse. If the node is in the sparse region, it retransmits the RREQ packet with high probability so that it can reach to maximum no. of nodes, otherwise it forwards the packet with a low probability, if node is in the dense network region. The new model is implemented in GloMoSim simulator [6] to perform a no. of simulations and performance of new IAODV protocol is compared and analyzed against the AODV[7] based on pure flooding algorithm.

\section{RELATED STUDY}

Williams and Camp [8] have classified the broadcast protocols into flooding, probability-based, counter based, Distance based, location-based and neighbor knowledge schemes. Similarly, neighbour knowledge schemes can be divided into selecting forwarding neighbours and clusteringbased. The counter-based scheme inhibits the rebroadcast if the packet has already been received for more than a given number of times.

N. Karthikeyan et. al [9] presented an overview of the broadcasting techniques in mobile ad hoc networks, The comparative study concludes that simple flooding requires each node to rebroadcast all packets. Probability based methods use some basic understanding of the network topology to assign a probability to a node to rebroadcast. Area based methods assume nodes have common transmission distances: a node will rebroadcast only if the rebroadcast will reach sufficient additional coverage area. Neighbor knowledge methods maintain state on their neighborhood via "Hello" packets, which are used in the decision to rebroadcast.

Abdalla M. Hanashi et al [10] proposed a dynamic probabilistic broadcasting scheme for mobile ad hoc networks where nodes move according to way point mobility model. The proposed approach dynamically sets the value of the rebroadcast probability for every host node according to the neighbor's information. The simulation results prove this approach can generate less rebroadcasts than that of the fixed 
probabilistic approach, while keeping the reachability high. It also demonstrates lower collisions than all the presented approaches.

W.R. Salem Jeyaseelan et al [11] presented a routing discovery strategy for mobile ad hoc networks. Performance of protocols with respect to scalability has also been analyzed. Results showed that, AODV and OLSR experienced higher packet delay and network load compared to DSR. This was due to the localization mechanism employed in DSR. On the other hand, when segment delay is considered both OLSR and AODV performed very reliably and established quick connection between nodes without any further delay.

Hussein Al-Bahadili et al [12] presented a detailed description of a simulation model that is developed to analyze the performance of a probabilistic algorithm for route discovery in noisy MANETs. Probabilistic broadcast has been widely used as a flooding optimization mechanism to alleviate the effect of broadcast storm problem (BSP) in mobile ad hoc networks (MANETs). Many research studies have been carried-out to develop and evaluate the performance of this mechanism in an error-free (noiseless) environment. In reality, wireless communication channels in MANETs are an error-prone and suffer from high packet-loss due to presence of noise, i.e., noisy environment.

In the probabilistic scheme [13] when receiving a broadcast packet for the first time, a node rebroadcasts the packet with a probability $\mathrm{p}$; when $\mathrm{p}=1$, this scheme reduces to blind flooding. In the distance-based scheme a node rebroadcasts the packet only if the distance between the sender and the receiver is larger than a given threshold. In the location-based scheme, a node rebroadcasts a packet only when the additional coverage due to the new emission is larger than a certain bound. In the selecting forwarding neighbours a broadcasting node selects some of its1-hop neighbours as rebroadcast nodes.

Kim et al. [14] proposed broadcasting scheme combines neighbor coverage and probabilistic based schemes in which a mobile host can dynamically adjust the value of rebroadcast probability according to its additional coverage in its neighborhood distance from the sender. This technique improves the results to a great distance but does not provide the optimal adaptation strategy to reduce overheads and increase reachability.

Kousha et al. [15] proposed a probability based algorithm to optimize broadcasting operation in MANET routing based on some intelligent form of gathering neighborhood information and transmission ranges by ensuring that instead of using common optimum transmission range a variable transmission range could be used to increase the capacity of the network. The work concluded with a inverse function relationship between neighborhood density and rebroadcast probability.

Dembla et al. [16] proposed an algorithm to reduce the broadcast storm problem based on a mechanism of combining probabilistic and neighbor density methods for better reachability and low overheads hence reduced collisions. This protocol dynamically adjusts the probability of rebroadcasting the route requests to its neighbors based on the density of nodes i.e. it is set high if nodes density region is sparse and vice-versa for better coverage in the same transmission range. Results show improvement in saved rebroadcast and better end to end delay etc.
Some more authors have proposed various algorithms [17][18][19][20] for controlled redundancy and efficient flooding, adaptive flooding in MANETs, etc. but all suffer from performance bottlenecks because of high broadcast messages in the network during route discovery phase of on demand routing protocols. Authors in [21] proposed a novel approach of broadcasting in MANETs which gives very good results in varying network density environments.

\section{PROPOSED IMPROVED (I-AODV) PROTOCOL \& ANALYSIS}

In pure flooding or simple flooding approach, a source node broadcasts it $\mathrm{s}$ packet to all neighbors. Each of those neighbors in turn rebroadcast the packet first time it receives the packet. Redundant packets are simply dropped. This behavior continues until all reachable network nodes have received. However, blind flooding produces high overhead in the network, resulting in the broadcast storm problem. In, a innovative method is proposed for reducing broadcast overhead in flooding based message delivery. This algorithm prevents blindly flooding requests packets in the whole network and involves a number of nodes in the request process to ensure route discovery rate and the transmission range is determined without considering the node distribution of the network hence may flooding zone may contain excess nodes and increases overheads which is not acceptable.

Here, we propose novel approach of dynamically adjusted flooding to yield higher performance. In addition, it is simple enough for easy implementation without the use of maintaining a counter for duplicate packets. In our research study we contribute to minimize the broadcast storm problem and propose a improved protocol based on a new method which is a new probabilistic approaches based on distance based selective flooding. In Distance based probabilistic broadcasting approach We use the distance of a node to estimate forwarding probability and adjust the rebroadcast probability. If a mobile node is located in the area closer to sender, its rebroadcast probability will be set lower. On the other hand, if a mobile node is located in the area far from sender, its rebroadcast probability will be set higher, because rebroadcast through this node can cover much extra area.

The proposed algorithm tries to avoid that situation by giving high priority at that point. Similarly, if a node is having high density of neighbors, then there will be lot of chance for packet collision at that point. The proposed algorithm tries to avoid that situation by giving low priority at that point. Network density demonstrates the node distribution of the local area. This algorithm is mix of probability and knowledge based approaches. It dynamically adjusts the RREQ probability of rebroadcasting at each node, as per the value of no. of neighbors and this value is large in sparse region as compared to dense regions. The decision to rebroadcast RREQ packets is made instantly after receiving a packet without any delay.

Simulation results show that our approach can improve the average performance of broadcasting in various network scenarios. A brief outline of the improved-AODV algorithm is as follows;

Begin

\{

Step 1: When abroadcast RREQ packet is received at a node $x$ for the first time;

Step2: Get the Number of neighbours for 1-hope 
i.e. local neighborhood information.

Step3: Get the Number of neighbours within node's

Transmission range.

Step4: Set the value of rebroadcast probability

according to local and global density:

( $P=$ NHello_Packet /Transmission_Range.)

Step4: Generate a random uniform number $R$ over the

interval

between $[0,1]$

I

If $(R>P)$

Rebroadcast the received RREQ packet

Else

Free $(R R E Q)$

\}

End

The rebroadcast probability should be set differently for one node to another in order to alleviate the number of rebroadcasting RREQ control packets and increase the efficiency of the network. Upon the selection of the value of forwarding $P$, the algorithm generates a random number between the interval $[0,1]$, compares it with the value of $P$, and decides to rebroadcast or drop the RREQ packet.

\section{EXPERIMENTAL SET-UP FOR PROTOCOL IMPLEMENTATION}

The performance is analyzed against parameters such as mobility and node density. The simulations are done in GloMoSim simulator. The necessary changes in the code of AODV protocol are done and modified code is simulated in the GloMoSim simulator. The objective is to reduce the no. of RREQ packets. i.e. reducing the no. of forwarding nodes, which would reduce the signal collision in the network. Both the protocols are simulated in same settings of parameters and scenarios to compare the results. Simulations are run on 4 seeds and the averaging of the values is used for final analysis and comparison.

The mobility model used is Random Waypoint Mobility model in a terrain range of $1000 \times 1000$ meters. According to this model, each node in the beginning of the simulation remains stationary for a pause time second, then chooses a random destination and starts moving towards it with a random speed [0, max.-speed] and after reaching at destination, node stops for a pause time interval and chooses a new destination and speed. This process repeats until the simulation ends.

To analyze the performance of AODV and protocols, scenarios are set as per the parameters shown in table 1 .

Table 1 Simulation environment parameters

$\begin{array}{ll}\text { SIMULATION-TIME } & 45 \mathrm{M} \\ \text { TERRAIN-DIMENSIONS } & (1000,1000) \\ \text { NUMBER-OF-NODES } & 25,50,100,150 \\ \text { NODE-PLACEMENT } & \text { RANDOM } \\ \text { MOBILITY } & \text { RANDOM } \\ \text { WAYPOINT } & \\ \text { MOBILITY-WP-PAUSE } & 30 \mathrm{~S} \\ \text { MOBILITY-WP-MIN-SPEED } & 0 \\ \text { MOBILITY-WP-MAX-SPEED } & 10,20,30,50(\mathrm{~m} / \mathrm{sec}) \\ \text { PROPAGATION-PATHLOSS } & \text { TWO-RAY } \\ \text { RADIO-FREQUENCY } & 2.4 \mathrm{e} 9 \\ \text { RADIO-BANDWIDTH: } & 2000000 \mathrm{bps} \\ \text { RADIO-TX-POWER: } & 15.0 \mathrm{dBm} \\ \text { MAC-PROTOCOL } & 802.11\end{array}$

\section{PERFORMANCE METRICS}

AODV and I-AODV are included in simulator for evaluating and comparing the performance of the protocols in various network densities, node distribution, mobility scenarios etc. It is assumed in all the simulations that links between all the nodes are bidirectional and transmission range is a circle area. The performance of broadcast protocols can be measured by a variety of metrics. A commonly used metric is the number of message retransmissions with respect to the number of nodes. In case of broadcasting with adjusted transmission power (thus adjusted disk that the message can reach), the total power can be used as performance metrics. The next important metric is reachability, or the ratio of nodes connected to the source that received the broadcast message.

Evaluation of the algorithm is done using following performance metrics:

- Routing overhead: It is the total number of route request packets transmitted during the simulation time. If there is multi hop transmission, each transmission over 1-hop is counted as 1-transmission.

- End to end delay: It is the average time difference between the time a data packet is sent by the source node and the time it is received by the destination node. This is average end to end delay of all successfully transmitted data packets from source to destination. Formula for average end to end delay is:

$$
\text { Average End to end Delay }=\frac{\sum_{1}^{n}(C B R \text { sent time }-C B R \text { receive time })}{\sum_{1}^{n} C B R \text { receive time }}
$$

where $\mathrm{n}$ is number of received packets.

- Throughput: It is the total no. of data packets received at the destination in one second.

- Average no. of Collisions: It is the total no. of packets dropped resulting from the collisions at the MAC layer.

- No. of Broadcasts: No. of broadcast packets sent across the network also affects the performance of the protocol in terms of bandwidth consumption and other overheads.

\section{RESULT ANALYSIS AND PERFORMANCE EVALUATION}

A few of the above mentioned metrics are evaluated for simulation set up of AODV and I-AODV protocols.

Routing Overhead Analysis: The routing overhead increases when the network density increases as shown in the figure 1. It is clear from the graph that the routing overhead reduces in as compared to AODV, at low and medium dense networks. The overheads in reduces to around $60 \%$ as compared to conventional AODV protocol. It increases proportionally with the increase in the no. of nodes as well as speed of the nodes also. There is a direct relationship between the no. of nodes in the network and the no. of RREQ packets. The routing overhead increase with the increase of traffic load also. When the density increases, no. of RREQs also increases and in turn no. of duplicate packets also increases which leads to more contention and collisions rate also. Similarly the no. of broadcasts packets increases with the increase in the speed, no. of nodes, and no. of source nodes. There is a relatively very less no. of broadcasts requests in protocol as compared to conventional AODV protocol, because there is now selective rebroadcasting based on node density at each node. It shows that I-AODV is more scalable than AODV in terms of higher node density in a fixed area. 


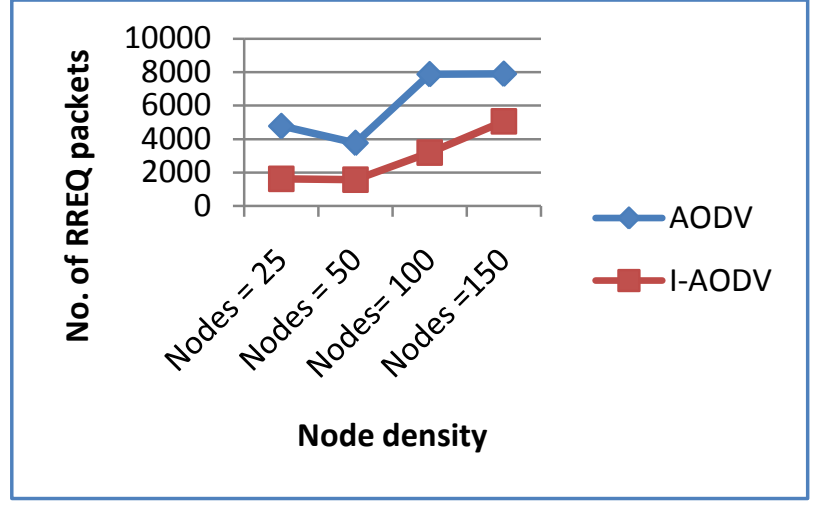

Fig. 1 : Node density Vs Routing overhead (no. of RREQ pkts.)

End to End Delay Analysis : It is the average time difference between the time a data packet is sent by the source node and the time it is received by the destination node. The fig. 2 clearly shows that there is around $10 \%$ to $20 \%$ less end to end delay in I-AODV protocol as compared to AODV protocol and this delay increases with the increase in speed because route paths between nodes change more frequently and more requests need to be performed. When a node receives the packet, it immediately decides whether to forward to the neighbor or discard the packet immediately, based on the local node density. When network density increases, more RREQ packets fail to reach the destinations due to high probability of packet collisions and channel contention caused by excessive redundant retransmissions of route requests packets.

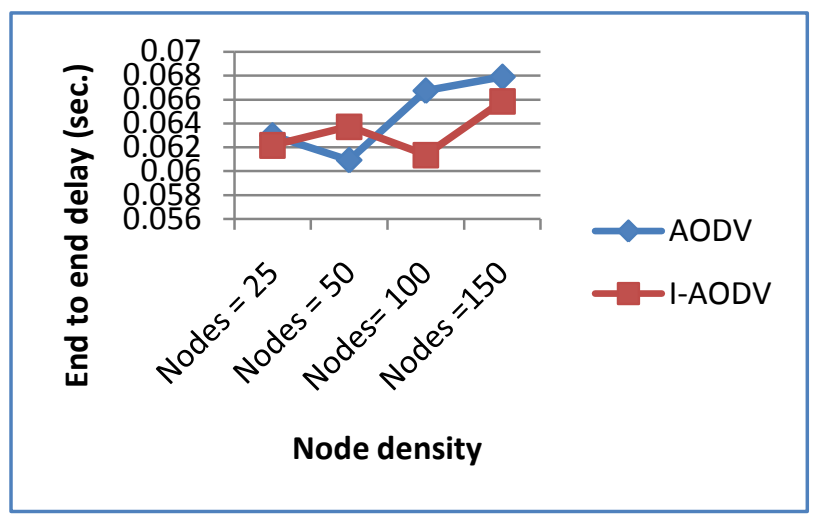

Figure 2. Node density Vs. End to End Delay

Collision Rate Analysis: Fig. 3 shows that the no. of packets collisions increases as the no. of nodes increase. The graph shows that there is around $30 \%$ less collisions in IAODV protocol as compared to AODV. The no. of collisions increase with the increase in no. of nodes and speed also. In IAODV large duplicate packets are reduced as the possibility of having more than 2 nodes transmitting at the same slot is reduced when the no. of nodes increases. When the no. of source nodes increases, generated no. of RREQ packets also increases. Hence, many RREQ packets collide with each other and due to contention among the nodes in shred transmission channel in the network.

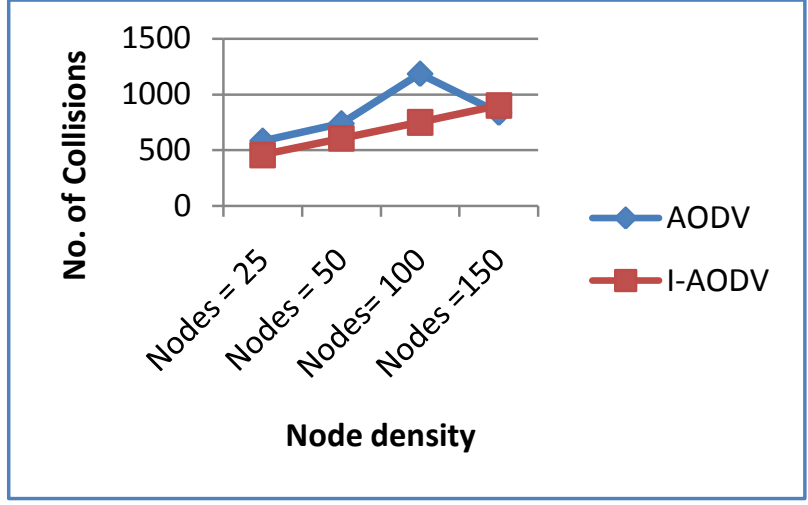

Fig. 3 : Node density Vs. No. of Collision

Broadcast Packets Sent Analysis : The total no. of broadcast packets sent over the network also adds on to the overhead and affects the performance of the network. It is also a good measure of the performance of the protocol. The total no. of broadcast packets sent in I-AODV protocol is around $40 \%$ to $50 \%$ less than that in AODV as shown in the fig. 4 . It increases exponentially with increase in the node density in a MANET scenario.

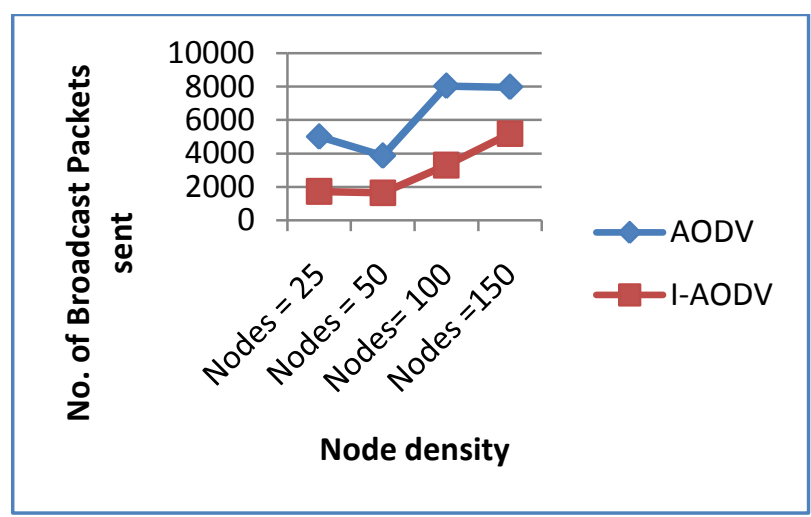

Figure 4. Node density Vs. No. of Broadcast pkts. Sent.

Throughput and packet delivery ratio also improves around $10 \%$ and 3 to $5 \%$ in case of I-AODV protocol as shown in figure 5 and figure 6 . The no. of control packets used also decreases to around $50 \%$ as shown in figure 7 .

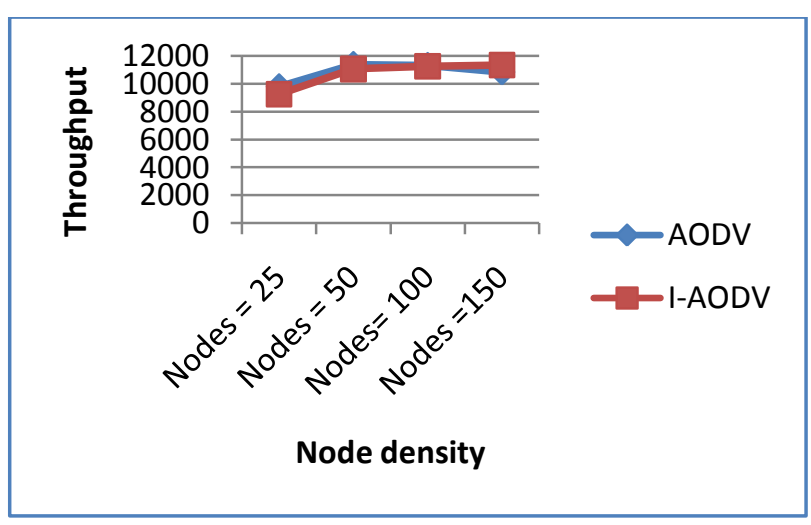

Figure 5. Node density Vs. Throughput. 

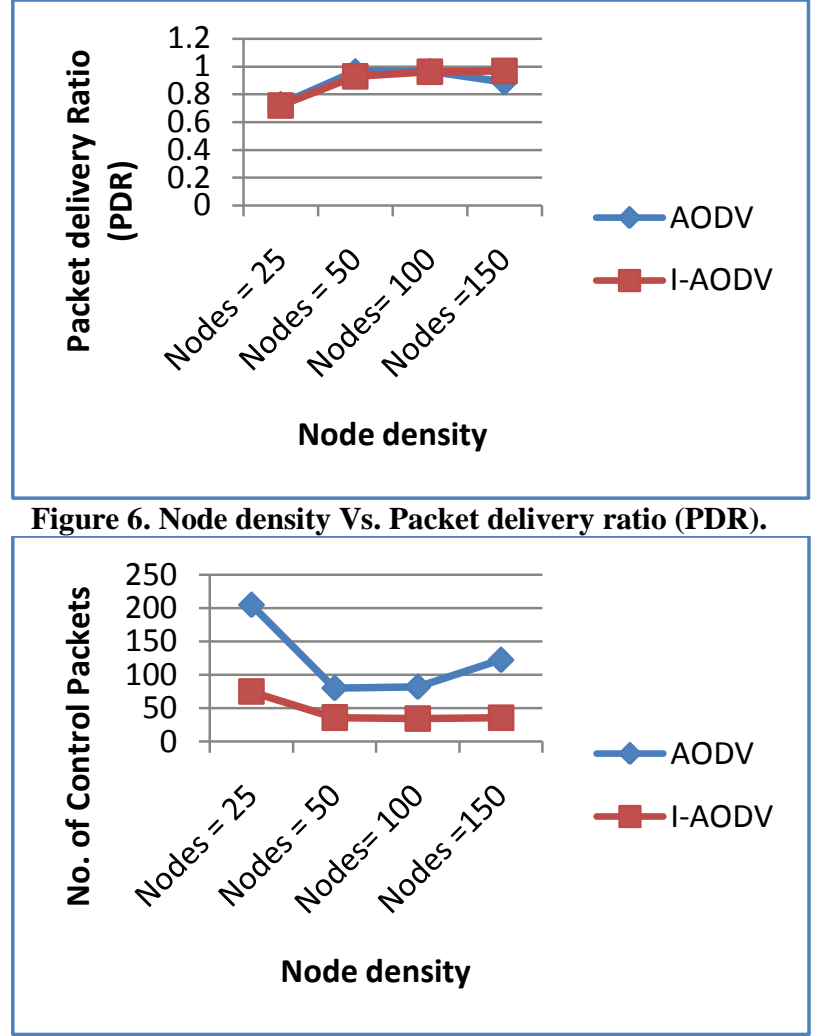

Figure 7. Node density Vs. No. of Control packets.

It is found from the analysis, that all the performance measures discussed above have been improved to a great extent in I-AODV protocol as compared to results of conventional AODV protocol based on pure flooding method. The results of this new improved protocol are also compared and found improved over by the previous work done on AODV by Rahman, W. Olesinski and P. Gburzynski in [22], W. Peng, X.C. Lu in [23] , The no. of broadcasts is minimum in I-AODV protocol which increases its efficiency in congested networks. The results confirm that I-AODV, which is based on smart route discovery method, performs better than conventional AODV which is based on simple flooding approach.

\section{CONCLUSION AND FUTURE SCOPE}

The main conclusion of this research work is that proposed IAODV protocol offers better performance than conventional AODV protocol which uses simple flooding for route discovery process. In blind flooding technique (used in AODV), each node in the network, retransmits the RREQ packet, resulting in the maximum no. of retransmissions. In our new approach, selective flooding is done, based on neighbor node density and probability of RREQ rebroadcasting. The reliability depends largely on the performance in the highly dynamic networks. The results confirm that, which is based on smart improved route discovery method, performs better than conventional AODV which is based on simple flooding approach. The performance is evaluated in terms of some important performance metrics such as end-to-end delay, routing overheads, control overheads, no. of collisions, no. of broadcast pkts etc., and is found better than AODV. The no. of broadcasts is min. in IAODV protocol which increases its efficiency in congested networks. In future work it is recommended to investigate the performance of the protocol in different mobility models etc. and on different other on demand routing protocols of MANETs such as DSR, TORA etc. to investigate their performance in similar scenarios. It is concluded from the results that the improved protocol I-AODV is superior to conventional AODV which implement a unique technique of combination of probability based and node density based technique to determine rebroadcast probability of route request retransmission in MANETs AODV routing protocol. and it controls the broadcast storm problem which is a performance bottleneck in AODV routing protocol in MANETs.

\section{REFERENCES}

[1] C-K. Toh, Ad Hoc Mobile Wireless Networks: Protocols and Systems, Prentice Hall PTR, 2002.

[2] Perkins C, Belding-Royer E, Das S. Ad hoc on-demand distance vector (AODV) routing. In: IETF mobile ad hoc networking Working Group INTERNET DRAFT, RFC 3561, July 2003, http://www.ietf.org/rfc/rfc3561.txt. Experimental RFC.

[3] Johnson D, Hu Y, Maltz D. The Dynamic Source Routing Protocol (DSR). In: IETF mobile ad hoc networking Working Group INTERNET RAFT, RFC 4728 , February 2007,http://www.ietf.org/rfc/rfc4728.txt

[4] S.-Y. Ni, Y.-C. Tseng, Y.-S. Chen, J.-P. Sheu, The broadcast storm problem in a mobile ad hoc network, Wireless Networks, vol. 8, no. 2, pp.153-167, 2002.

[5] Rahman, W. Olesinski and P. Gburzynski. Controlled Flooding in Wireless Ad Hoc Networks. Proceedings of IEEE International Workshop on Wireless Ad Hoc Networks (IWWAN'04), University of Oulu, Finland, 2004.

[6] Jorge Nuevo, "A Comprehensible GloMoSim Tutorial”, University of Quebec, September 2003

[7] C.E. Perkins and E.M. Royer, "Ad-hoc on-demand distance vector routing," in Proceedings of the Second Annual IEEE Workshop on Mobile Computing Systems and Applications, Feb. 1999, pp. 90-100.

[8] T. Camp, J. Boleng, B. Williams, L. Wilcox, and W. Navidi. Performance evaluation of two location based routing protocols. In Proceedings of INFOCOM 2002.

[9] N. Karthikeyan et al. "Performance Comparison of Broadcasting methods in Mobie Ad Hoc Network", In proceedings of International Journal of Future Communications and Networking, Vol. 2. No. 2 June, 2009. Pp. 47-58.

[10] Abdalla M. Hashmi et al. , "Improvin Route Discovery of Dynamic Probabilistic Flooding in On-Demand Routing Protocols for MANETs", in Proceedings of $10^{\text {th }}$ Telecommunications Forum, 2008. Pp. 89-92.

[11] W.R S. Jeyaselan et al. , "Investigation on Routing Protocols in MANET", In International Journal of research and reviews in Information Sciences, Vol. 1. No.2, June 2011.

[12] Hussein Al-Bahadili1 and Rami Jaradat, "Performance Evaluation of an OMPR Algorithm for Route Discovery in Noisy MANETs", in International Journal of Computer Networks \& Communications (IJCNC), Vol. 2, No. 1, January 2010, pp. 85-96.

[13] Qi Zhang and D.P. Agrawal, "Analysis of Leveled Probabilistic Routing in Mobile ad hoc Networks", Communications, 2004 IEEE International Conference on, 7:pp. 3896-3900 7, June 2004.

[14] Jae-soo Kim et al. , "Probabilistic Broadcasting Based on Coverage Area and Neighbor Confirmation in Mobile Ad Hoc Networks", IEEE Communications Society Globecom 2004 Workshops. 
[15] Kousha et al. , “Low Interference Topology Control for Wireless Ad Hoc Networks", work supported by NSF CCR-0311174. Illinois Institute of technology, Chicago.

[16] Deepak Dembla et al. , "Modeling and Analysis of an Intelligent AODV Routing Protocol based on Route Request Retransmission Strategy in MANETs", www.ijcaonline.org/archives/volume30/number11/3684 -5190 .

[17] Y.-C. Tseng, S.-Y. Ni, and E.-Y. Shih, "Adaptive Approaches to Relieving Broadcast Storms in a Wireless Multihop Mobile Ad Hoc Network," IEEE Trans. Computers, vol. 52, no. 5, pp. 545-557, May2003.

[18] J.Wu and W. Lou,"Forward-node-set-based broadcast in clustered mobile ad hoc networks, "Wireless Communications and Mobile Computing, vol. 3, pp. 155-173, 2003.

[19] H. Lim and C. Kim, "Flooding in Wireless Ad Hoc Networks," In Proceedings of ACM MSWiM Workshop at MobiCom, Aug. 2000; Computer Communication Journal, 24, 3-4, 353-363, 2001.
[20] Al-rodhan et al. "Efficient Route Discovery Algorithm for MANETs", In proceedings of $5^{\text {th }}$ IEEE International Conference on Networking, Architecture and Storage, 2010, 165-170.

[21] M. K Yadav et al., "A Novel Approach of Broadcasting in AODV Routing Protocol in MANETS", In International Journal of Electronics Engineering, 3 (1), 2011, pp. 145149.

[22] Rahman, W. Olesinski and P. Gburzynski. Controlled Flooding in Wireless Ad Hoc Networks. Proceedings of IEEE International Workshop on Wireless Ad Hoc Networks (IWWAN'04), University of Oulu, Finland, 2004.

[23] W. Peng, X.C. Lu, on the reduction of broadcast redundancy in mobile ad hoc networks, in: Proc. Workshop on Mobile and Ad Hoc Networking and Computing (MobiHOC'2000), Boston, Massachusetts, $\begin{array}{llll}\text { USA, } & \text { p } . & 129-130,\end{array}$ 